\title{
Three-Dimensional Acoustic Double-Zero-Index Medium with a Fourfold Degenerate Dirac-like Point
}

\author{
Changqing Xu, ${ }_{1}^{1}$ Guancong Ma, ${ }^{2, *}$ Ze-Guo Chen, ${ }^{1,2}$ Jie Luo, ${ }^{3,4}$ Jinjie Shi, ${ }^{4}$ Yun Lai, ${ }^{4, \dagger}$ and Ying $\mathrm{Wu}^{1, \$}$ \\ ${ }^{1}$ Division of Computer, Electrical and Mathematical Science and Engineering, \\ King Abdullah University of Science and Technology (KAUST), Thuwal 23955-6900, Saudi Arabia \\ ${ }^{2}$ Department of Physics, Hong Kong Baptist University, Kowloon Tong, Hong Kong \\ ${ }^{3}$ School of Physical Science and Technology, Soochow University, Suzhou 215006, China \\ ${ }^{4}$ MOE Key Laboratory of Modern Acoustics, National Laboratory of Solid State Microstructures, School of Physics, \\ and Collaborative Innovation Center of Advanced Microstructures, Nanjing University, Nanjing 210093, China
}

(Received 14 April 2019; accepted 15 January 2020; published 19 February 2020)

\begin{abstract}
We report the first realization of a three-dimensional (3D) acoustic double-zero-index medium (DZIM) made of a cubic lattice of metal rods. While the past decade has seen several realizations of 2D DZIM, achieving such a medium in 3D has remained an elusive challenge. Here, we show how a fourfold degenerate point with conical dispersion can be induced at the Brillouin zone center, such that the material becomes a 3D DZIM with the effective mass density and compressibility simultaneously acquiring nearzero values. To demonstrate the functionalities of this new medium, we have fabricated an acoustic waveguide of 3D DZIM in form of a "periscope" with two $90^{\circ}$ turns and observed tunneling of a normally incident planar wave through the waveguide yielding undistorted planar wave front at the waveguide exit. Our findings establish a practical route to realize 3D DZIM as an effective acoustic "void space" that offers unprecedented control over acoustic wave propagation.
\end{abstract}

DOI: 10.1103/PhysRevLett.124.074501

Wave propagating in a medium with one or more constitutive parameters vanishing does not accumulate any phase retardation. This characteristic can be leveraged to achieve wave manipulation in unique ways, enabling unctionalities like wave front engineering [1-11], cloaking of objects [9-16], wave tunneling [17-25], asymmetric transmission [26-28], and photonic doping [29-34]. A single-zero-index medium, with only one constitutive parameter near zero, usually has a significant impedance mismatch with the background, which is undesirable for real applications. A double-zero-index medium (DZIM), with both constitutive parameters near zero, can overcome this obstacle owing to its finite effective impedance [8-12]. In the past decade, several approaches have been successfully utilized to realize the two-dimensional (2D) DZIM, including Dirac-like conical dispersion [8-11,20,24], the doping of single-zero-index media [29-34], and the use of parity-time symmetry [35].

Despite these accomplishments, the applicability of 2D DZIMs is intrinsically limited to the control of in-plane propagating waves $[5,14]$. Three-dimensional (3D) DZIMs would allow overcoming this constraint and achieving far superior functionalities. The ascension of dimensionality not only satisfies the critical need of wave manipulation but also leads to fundamental new physics. However, from two to three dimensions, we face a multitude of difficulties. For electromagnetic waves, the widely adopted strategy in two dimensions, i.e., the degeneracy of dipolar and monopolar modes [4,7-11], is ineffective because the transverse nature prevents the existence of a monopolar mode in three dimensions. For acoustic waves, although monopolar modes exist in 3D, natural slow-wave materials, typically chosen for scatterers, are extremely limited for airborne sound [6]. These differences and constraints hinder the extension of 2D DZIM to three dimensions in both electromagnetic and acoustic systems, leaving the realization of 3D DZIM a long-standing challenge. Recently, there were several theoretical attempts [36-38], which are either nonrealistic or rely on resonances that are sensitive to the intrinsic loss of the material.

Here, we report the first realization of a 3D DZIM for airborne sound. To overcome the aforementioned difficulties, instead of relying on previous approaches, we exploit the combination of glide symmetry [39-42] and accidental degeneracy, and design a phononic crystal (PC) that supports the accidental degeneracy of threefold degenerate dipolar states and a monopolar state at the Brillouin zone center. In the vicinity of this fourfold degenerate point, linear dispersion exists in all directions of the reciprocal space. We call it a Dirac-like point in the 3D $k$ space and find the effective mass density and compressibility are simultaneously zero at its frequency [43,44]. Although occupying a finite volume, the DZIM inherits the wave properties of a $3 \mathrm{D}$ effective void space, leading to unusual physics and applications. By experimentally constructing the 3D acoustic DZIM and putting it inside a twisted 


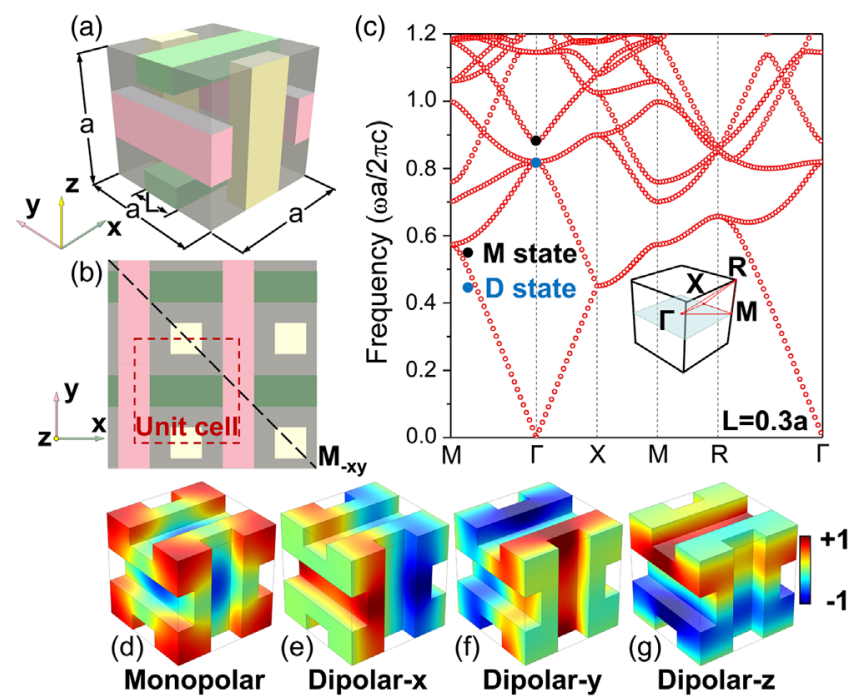

FIG. 1. Band structure and eigenstates of the 3D PC. (a) 3D view and (b) (001) top view of unit cell. The host medium (gray) is air. Colored square blocks with side length $L \times L \times a$ are aluminum blocks. The black dashed line and red dashed box in (b) represents the direction of mirror plane $M_{-x y}$ and the unit cell shown in (a). (c) Band structure of the PC with $L=0.3 a$. Black and blue dots represent the monopolar state and the threefold degenerate dipolar states at the Brillouin Zone center, respectively. (d)-(g) Acoustic pressure field distributions of the monopolar state and dipolar states.

acoustic periscope, we demonstrate unusual wave tunneling functionality with a nondisturbed planar wave front at the exit, which is a direct proof of the double-zero-index property in three dimensions.

As illustrated in Fig. 1(a), the PC is a cubic lattice of unit cells, each comprising three aluminum rods (treated as sound-hard objects) in air. The lattice constant is $a$. The aluminum rods are orthogonally aligned along the $x, y$, and $z$ directions, respectively, all having a square cross section with a side length $L$. When the axes of the rods are separated by $a / 2$, the system has a glide symmetry [39-42] defined as $G_{i j}=\left\{M_{-i j} \mid(a / 2) \hat{k}\right\}:(i, j, k) \rightarrow(-j,-i, k+a / 2)$, transforming the $i$ th $(i=x, y, z)$ rod into the $j$ th $(j=y, z$, $x$ ) rod. The lattice also has the $C_{3}$ rotational symmetry about the [111] diagonal axes [45] and mirror symmetry about the $x-y, y-z, z-x$ planes as shown in Figs. 1(a) and 1(b). The band structure of the PC with $L=0.3 a$ is shown in Fig. 1(c), exhibiting a threefold degeneracy at the $\Gamma$ point for the second, third, and fourth branches. The eigenfields of these degenerate states and a single state on the fifth branch are plotted in Figs. 1(d)-1(g), showing the single state [Fig. 1(d)] a monopolar state and the threefold degenerate states [Figs. 1(e)-1(g)] three orthogonal dipolar states. The degeneracy of these dipolar states is guaranteed by the symmetry of the lattice, regardless of the detailed geometric parameters. Altering the size of the rods only changes the eigenfrequency of these states. Therefore, (a)
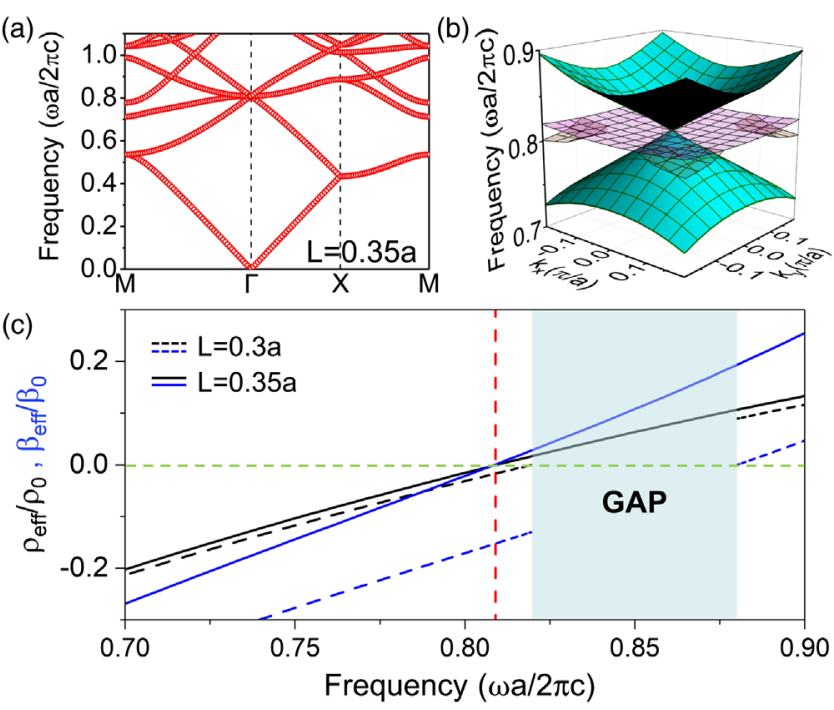

FIG. 2. Realization of 3D acoustic DZIM by accidental degeneracy. (a) Band structure of the PC with $L=0.35 a$. A fourfold degenerate point (the Dirac-like point) at the $\Gamma$ point is observed. (b) An enlargedplot of the dispersion surfaces near the Dirac-like point in the $k_{x}-k_{y}$ plane. (c) The normalized effective mass density $\rho_{\text {eff }}$ (black curves) and compressibility $\beta_{\text {eff }}$ (blue curves) for the cases of $L=0.35 a$ (solid curves) and $L=0.3 a$ (dashed curves).

accidental degeneracy of the monopolar and dipolar states, i.e., a fourfold degenerate point, can be achieved by changing $L$.

Figure 2(a) shows the band structure of a PC with $L=0.35 a$. The aforementioned fourfold degenerate point at the Brillouin zone center is observed. An enlarged view of the band structure near the $\Gamma$ point in the $k_{x}-k_{y}$ plane is shown in Fig. 2(b), demonstrating a conical dispersion intersecting with two flatbands. The dispersions in the $k_{y}-k_{z}$ and $k_{z}-k_{x}$ planes are the same as that in the $k_{x}-k_{y}$ plane [46], indicating linear dispersion emerges in the vicinity of this fourfold degenerate point in all directions. We call this point a Dirac-like point in the 3D $k$ space.

The Dirac-like point found here is different from the widely studied Dirac points and Weyl points, despite some similarities. Although both Dirac and Dirac-like points exhibit linear dispersion, only the Dirac points can be mapped into the massless Dirac Hamiltonian and carry a nonzero Berry phase [49-51]. The Weyl points always emerge in pairs, and possess opposite topological charges [52-57]. The topological characteristics of Weyl points have been explored for topological surface states [5456,58,59], negative refraction [54], etc. The Dirac-like point, on the other hand, possesses unique properties to be revealed later.

The Dirac-like linear dispersion discussed here also appears as a consequence of double-zero effective parameters [46]. In Fig. 2(c), we show the effective mass density $\rho_{\text {eff }}$ and compressibility $\beta_{\text {eff }}$ for our PC obtained from the 
effective parameter retrieval method based on field averaging $[43,44]$. For the case of $L=0.35 a$, both $\rho_{\text {eff }} / \rho_{0}$ and $\beta_{\text {eff }} / \beta_{0}$ cross zero simultaneously at the frequency of the Dirac-like point $\omega_{D}=0.807(2 \pi c / a)$. In comparison, when $L=0.3 a$, the effective mass density and compressibility cross zero at different frequencies $\omega_{d}=0.82(2 \pi c / a)$ and $\omega_{m}=0.88(2 \pi c / a)$, which correspond to the eigenfrequencies of the dipolar and monopolar states at the $\Gamma$ point, respectively. Therefore, only single-zero-index media are realized. When the band gap between $\omega_{d}$ and $\omega_{m}$ closes, the Dirac-like conical dispersion emerges as a result of the double-zero-index. Such results consist with the scattering theorem, in which the monopolar and dipolar resonances correspond to the effective bulk modulus and mass density, respectively [60]. We note that the effective medium approximation works well at this relatively high frequency because the effective wavelength approaches infinity, as has been physically explained in [61] and observed in previous 2D DZIM systems [7-9].

The additional flatbands in Figs. 2(a) and 2(b), with eigenstates shown in Figs. 1(f) and 1(g), correspond to zero effective mass induced transverse acoustic waves [46,60], which are deaf bands and couple weakly even to oblique incident waves. Therefore, their existence does not affect the wave properties near the Dirac-like point.

With vanishing $\rho_{\text {eff }}$ and $\beta_{\text {eff }}$, the 3D DZIM can be regarded as an acoustic void space. To demonstrate this unique property that is not available for any other acoustic materials, we consider the transmission of a normally incident wave through a slab of 3D DZIM with finite thickness. The transmittance is always unity, regardless of any potential impedance mismatch between the DZIM and the background, meaning that the incident wave passes through DZIM as if it was a void. We verify this property both analytically through the effective medium and numerically via simulation of the PC structure [46]. In comparison, a slab of an ordinary medium with mismatched impedance to the background always causes reflection unless Fabry-Perot resonances occur. The transmission coefficient of a slab of single-zero-index medium keeps at low values [46]. Indeed, our DZIM approximation is effective for small incident angles when the nonlocality effect is not significant. A 2D DZIM is equivalent to a "void plane" only for in-plane propagating waves [5-11,14]. A 3D DZIM of finite volume offers more possibilities. For example, a wave incident normally onto one surface of a DZIM cube is partitioned and radiates normally from the other five surfaces. If any four outlet surfaces are covered with totally reflective boundaries, the DZIM cube becomes a wave-steering device that can direct waves to orthogonal directions in all three dimensions with a $100 \%$ efficiency.

To demonstrate the potential for practical use of the 3D DZIM as a 3D acoustic void space, we have designed and fabricated a 3D periscope for sound. Figure 3(a) shows an acoustic bending waveguide filled with 3D DZIM. The

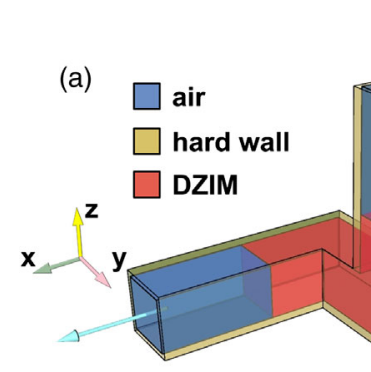

(c)
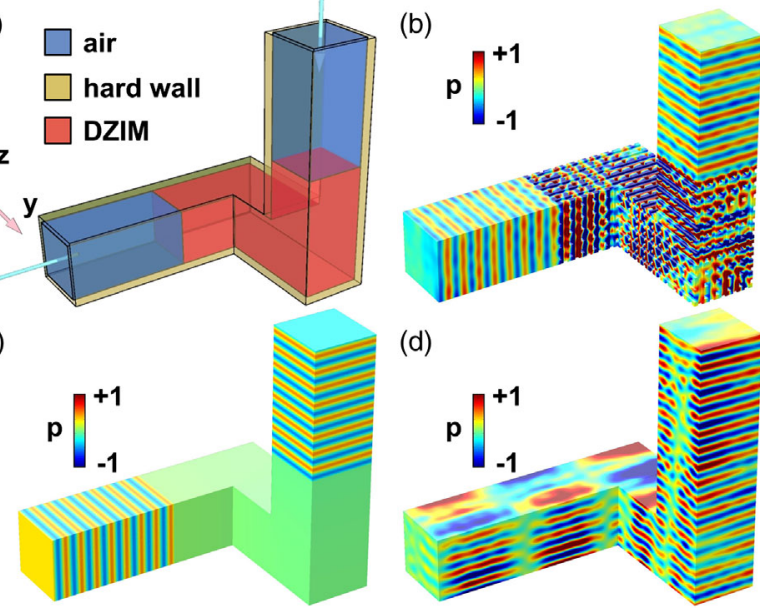

(d)

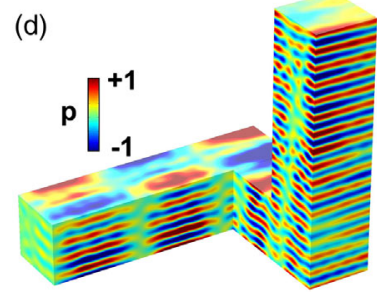

FIG. 3. Transmission of sound through an acoustic "periscope." (a) Illustration of the designed acoustic periscope consisting of a bending waveguide with two $90^{\circ}$ bends (gold color). The red and blue regions represent the 3D DZIM and air, respectively. Wave incident from the top. (b) Simulated pressure field distribution when the waveguide is filled with $\mathrm{PC}$ at $f_{\text {Dirac }}=9742 \mathrm{~Hz}$. (c) The same as (b) but the PC is replaced by its effective medium. (d) The same as (b) and (c), but without any filling.

wave comes from the top along the negative $z$ direction, impinging normally to the DZIM. With two $90^{\circ}$ bends, the waveguide turns into negative $y$ direction then to positive $x$ direction. Figures 3(b) and 3(c) show the simulated pressure field distribution with the filling material being the PC and its effective medium, respectively, at the Diraclike point frequency. For both cases, the systems have minimal reflection. Eigenstates away from the $\Gamma$ point at this frequency are not excited by normally incident waves because of their nonzero wave vector in the $k_{x}-k_{y}$ plane. Scattering by the bending parts of the waveguide is also weak, due to the large effective wavelength attributed to the near-zero index. As a result, the outgoing waves retain a planar wave front, which are reoriented and parallel to the $y-z$ plane. In contrast, without the DZIM, the wave is inevitably scattered by the bends that severely scrambles the outgoing wave front, as shown in Fig. 3(d). These results clearly demonstrate the equivalence of our PC and a 3D DZIM by wave-tunneling through a 3D bending waveguide with wave front-preserving reorientation, which is a direct and unique consequence of the 3D DZIM as an effective acoustic void space. Without the ascension of dimensionality in the DZIM, it is not possible to realize such a flexible bending waveguide that route waves in the 3D space.

Figure 4(a) shows the experimental setup of periscope waveguide with 12 aluminum plates. The waveguide has a square cross section with a side length of $14.25 \mathrm{~cm}$ (5 unit cells). As shown in Fig. 4(b), the PC is built inside the waveguide using aluminum rods with square cross sections 

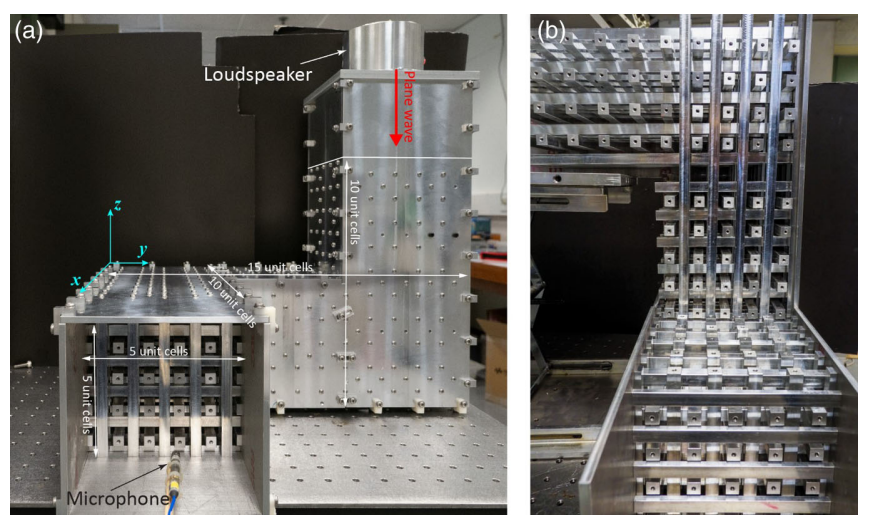

FIG. 4. Experimental setup. (a) A loudspeaker emits a plane wave along the negative $z$ direction. The waveguide bends twice and the output is along the positive $x$ direction. A microphone is mounted on a translational stage to scan the sound at the outlet in $y-z, x-y$, and $x-z$ planes. (b) Some boundaries of the waveguide are removed to show the PC inside.

of $1.0 \mathrm{~cm} \times 1.0 \mathrm{~cm}$. The lattice constant is $2.85 \mathrm{~cm}$. The simulation yields a Dirac-like point at $f_{\text {Dirac }}=\omega_{D} /(2 \pi)=$ $9742 \mathrm{~Hz}$. Around this frequency, the thicknesses of viscous boundary layer and thermal boundary layer are about 0.021 and $0.026 \mathrm{~mm}$, respectively, which are orders of magnitude smaller than the minimum separation of square rods, i.e., $4.25 \mathrm{~mm}$, meaning the viscothermal effect is negligible $[62,63]$. We mount a loudspeaker with a diameter of $12 \mathrm{~cm}$ on one end of the waveguide to generate a plane wave that propagates in the negative $z$ direction, as shown in Fig. 4(a). The transmitted wave at the exit of the waveguide is measured by a microphone, which is mounted on a translational stage to raster scan the wave profile in the $y-z, x-y$, and $x-z$ planes.

We perform a 2D Fourier transform of the spatial maps at frequencies near $f_{\text {Dirac }}=9680 \mathrm{~Hz}$. The Fourier transforms of the $y-z$ plane, which is parallel to the PC-air interface at the exit of the waveguide, are shown in Fig. 5(a). Near $f_{\text {Dirac }}$, the Fourier coefficients exhibit a spike at $k_{y}=k_{z}=0$. This implies that the acoustic wave exiting from the PC has a wave vector dominated by $k_{x}$, indicating a planar wave front with minimal distortion is maintained although the propagation direction has changed twice. For frequencies above and below $f_{\text {Dirac }}$, the outgoing waves possess $k_{y}$ and $k_{z}$ components, as the sharp peak gradually
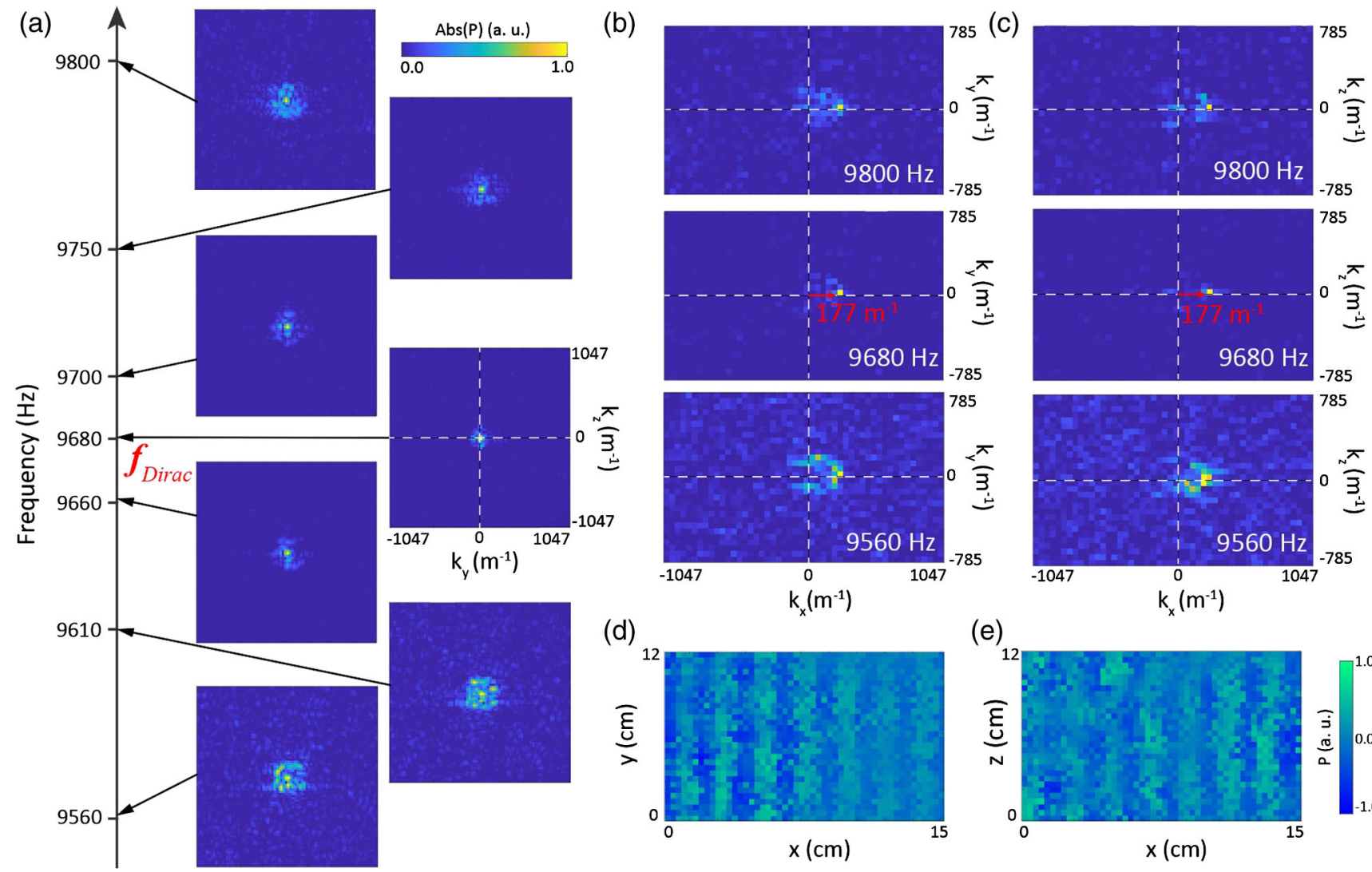

(e)

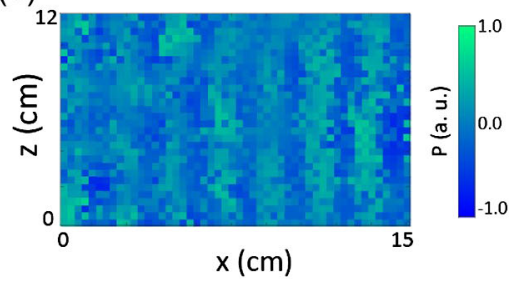

FIG. 5. Plane wave tunneling. (a) The 2D Fourier transforms of the scanned field patterns at frequencies near $f_{\text {Dirac }}=9680 \mathrm{~Hz}$. In the $k_{y}-k_{z}$ plane, the Fourier transform has a distribution that peaks at $k_{y}=k_{z}=0$ at $f_{\text {Dirac }}$, the distribution gradually expands as the frequency moves away from $f_{\text {Dirac }}$. All maps in (a) share the same coordinates. (b),(c) The same as (a) but for $k_{x}-k_{y}$ and $k_{x}-k_{z}$ planes. At $f_{\text {Dirac }}$ (middle panels), the Fourier transforms imply a plane wave in the positive $x$ direction. The red arrows mark the position of $k_{x}=2 \pi f_{\text {Dirac }} / c=177 \mathrm{~m}^{-1}, k_{y}\left(k_{z}\right)=0$. Away from $f_{\text {Dirac }}$, nonzero $k_{y}\left(k_{z}\right)$ components appear. (d) and (e) Scanned real-space field patterns of $x-y$ plane and $x-z$ plane at $f_{\text {Dirac }}$, respectively, showing a planar wave front. 
expands into a circle [Fig. 5(a)]. We further examine the 2D Fourier transforms of the rasterized map in the $x-y$ and $x-z$ planes, as shown in Figs. 5(b) and 5(c). At $f_{\text {Dirac }}$, the magnitudes sharply peak at $k_{x}=2 \pi f_{\text {Dirac }} / c \approx 177 \mathrm{~m}^{-1}$, clearly suggesting the outgoing wave is a plane wave. When the frequency deviates from $f_{\text {Dirac }}$, the wave starts to possess $k_{y}$ and $k_{z}$ components. In addition, the results obtained at 9800 and $9560 \mathrm{~Hz}$ are noticeably noisier than at $f_{\text {Dirac }}$, implying a reduction in the transmission coefficient away from $f_{\text {Dirac }}$. In Figs. 5(d) and 5(e), we plot the real-space map of the pressure fields at $f_{\text {Dirac }}$ in the $x-y$ and $x-z$ planes. Despite the presence of some noise, which is unavoidable due to the nonideal acoustic condition of our laboratory, well-defined planar wave fronts are detected, unambiguously showing that the sound remains a plane wave after bending twice in the waveguide. These experimental results convincingly demonstrate that at normal incidence, a plane wave at $f_{\text {Dirac }}$ can pass through the PC and be redirected into another direction with minimal distortion. This effect is the signature of a DZIM. We note that the measured $f_{\text {Dirac }}(9680 \mathrm{~Hz})$ deviates from the prediction $(9742 \mathrm{~Hz})$ by only $\sim 0.6 \%$ and is well within the fabrication tolerance. These results clearly demonstrate the equivalence of our PC and a 3D DZIM. Our work is not just about achieving the "acoustic periscope" that is difficult to realize for the frequency range we are interested in [46], but an important step forward in the realization of 3D DZIM that may benefit more practical applications.

In conclusion, we have for the first time demonstrated theoretically and realized experimentally a 3D DZIM using PCs. Because of the combination of lattice symmetry and accidental degeneracy, the PC exhibits a Dirac-like point at the Brillouin zone center in the 3D $k$ space. At the frequency of this point, the effective mass density and compressibility of the PC approaches zero simultaneously. Such rare property bestows on PCs intriguing functionalities of an effective acoustic void space that enables a nearly perfect sound tunneling through a suitably shaped 3D waveguide. Based on this recipe, we built the first working 3D DZIM in the form of an acoustic periscope bending waveguide filled with the PC and demonstrated that at the Dirac-like point frequency the sound can tunnel through sharp bends while maintaining a planar wave front. We have thus not only shown that the concept of DZIM can be extended to three dimensions, but also demonstrated that it can serve as a novel platform enabling unprecedented manipulation of sound in 3D.

We thank Dunjian Wang and Pengqi Li for assisting with the experiments, Professor C. T. Chan and Dr. Ruoyang Zhang for helpful discussions, Dr. Aigars Ekers for advice on the presentation, and anonymous referees for their valuable comments and suggestions. The work described here was partially supported by the King Abdullah University of Science and Technology (KAUST) Office of Sponsored Research (OSR) under Grant No. OSR-2016-
CRG5-2950 and KAUST Baseline Research Fund BAS/1/1626-01-01. G. M. was supported by the Hong Kong Research Grants Council (Grants No. RGC-ECS 22302718 and No. GRF 12300419), the National Natural Science Foundation of China (Grant No. 11802256), and by the Hong Kong Baptist University through FRG2/17-18/ 056, RC-SGT2/18-19/SCI/006. Y. L. was supported by the National Key R\&D Program of China (2017YFA0303702) and the National Natural Science Foundation of China (Grants No. 61671314, No. 11974176, and No. 11634005).

\footnotetext{
*phgcma@hkbu.edu.hk

†laiyun@nju.edu.cn

†Ying.Wu@kaust.edu.sa
}

[1] I. Liberal and N. Engheta, Near-zero refractive index photonics, Nat. Photonics 11, 149 (2017).

[2] H. Suchowski, K. O'Brien, Z. J. Wong, A. Salandrino, X. Yin, and X. Zhang, Phase mismatch-free nonlinear propagation in optical zero-index materials, Science 342, 1223 (2013).

[3] A. Alu, M. G. Silveirinha, A. Salandrino, and N. Engheta, Epsilon-near-zero metamaterials and electromagnetic sources: Tailoring the radiation phase pattern, Phys. Rev. B 75, 155410 (2007).

[4] P. Moitra, Y. Yang, Z. Anderson, I. I. Kravchenko, D. P. Briggs, and J. Valentine, Realization of an all-dielectric zeroindex optical metamaterial, Nat. Photonics 7, 791 (2013).

[5] M. Dubois, C. Shi, X. Zhu, Y. Wang, and X. Zhang, Observation of acoustic Dirac-like cone and double zero refractive index, Nat. Commun. 8, 14871 (2017).

[6] J. Hyun, W. Choi, S. Wang, C.-S. Park, and M. Kim, Systematic realization of double-zero-index phononic crystals with hard inclusions, Sci. Rep. 8, 7288 (2018).

[7] Y. Li, S. Kita, P. Muñoz, O. Reshef, D. I. Vulis, M. Yin, M. Lončar, and E. Mazur, On-chip zero-index metamaterials, Nat. Photonics 9, 738 (2015).

[8] F. Liu, X. Huang, and C. T. Chan, Dirac cones at $k=0$ in acoustic crystals and zero refractive index acoustic materials, Appl. Phys. Lett. 100, 071911 (2012).

[9] X. Huang, Y. Lai, Z. H. Hang, H. Zheng, and C. T. Chan, Dirac cones induced by accidental degeneracy in photonic crystals and zero-refractive-index materials, Nat. Mater. 10, 582 (2011).

[10] C. Xu and Y. Lai, Configurable Dirac-like conical dispersions in complex photonic crystals, Phys. Rev. B 95, 045124 (2017).

[11] F. Liu, Y. Lai, X. Huang, and C. T. Chan, Dirac cones at $k=0$ in phononic crystals, Phys. Rev. B 84, 224113 (2011).

[12] J. Luo, W. Lu, Z. Hang, H. Chen, B. Hou, Y. Lai, and C. T. Chan, Arbitrary Control of Electromagnetic Flux in Inhomogeneous Anisotropic Media with Near-Zero Index, Phys. Rev. Lett. 112, 073903 (2014).

[13] J. Luo, Z.-H. Hang, C. T. Chan, and Y. Lai, Unusual percolation threshold of electromagnetic waves in doublezero medium embedded with random inclusions, Laser Photonics Rev. 9, 523 (2015).

[14] Y. Li, K.-J. Zhu, Y.-G. Peng, W. Li, T. Yang, H.-X. Xu, H. Chen, X.-F. Zhu, S. Fan, and C.-W. Qiu, Thermal 
meta-device in analogue of zero-index photonics. Nat. Mater. 18, 48 (2019).

[15] L.-Y. Zheng, Y. Wu, X. Ni, Z.-G. Chen, M.-H. Lu, and Y.-F. Chen, Acoustic cloaking by a near-zero-index phononic crystal, Appl. Phys. Lett. 104, 161904 (2014).

[16] S. A. Cummer, J. Christensen, and A. Alù, Controlling sound with acoustic metamaterials, Nat. Rev. Mater. 1, 16001 (2016).

[17] R. Fleury and A. Alu, Extraordinary Sound Transmission through Density-Near-Zero Ultranarrow Channels, Phys. Rev. Lett. 111, 055501 (2013).

[18] M. G. Silveirinha and N. Engheta, Tunneling of Electromagnetic Energy through Subwavelength Channels and Bends Using $\varepsilon$-Near-Zero Materials, Phys. Rev. Lett. 97, 157403 (2006).

[19] B. Edwards, A. Alù, M. E. Young, M. G. Silveirinha, and N. Engheta, Experimental Verification of Epsilon-NearZero Metamaterial Coupling and Energy Squeezing Using a Microwave Waveguide, Phys. Rev. Lett. 100, 033903 (2008).

[20] Y. Li, Y. Wu, and J. Mei, Double Dirac cones in phononic crystals, Appl. Phys. Lett. 105, 014107 (2014).

[21] A. M. Mahmoud and N. Engheta, Wave-matter interactions in epsilon-and-mu-near-zero structures, Nat. Commun. 5, 5638 (2014).

[22] J. S. Marcos, M. G. Silveirinha, and N. Engheta, $\mu$-near-zero supercoupling, Phys. Rev. B 91, 195112 (2015).

[23] A. Alù, M. G. Silveirinha, and N. Engheta, Transmissionline analysis of $\varepsilon$-near-zero-filled narrow channels, Phys. Rev. E 78, 016604 (2008).

[24] Y. Li and J. Mei, Double Dirac cones in two-dimensional dielectric photonic crystals, Opt. Express 23, 12089 (2015).

[25] R. Liu, Q. Cheng, T. Hand, J. J. Mock, T. J. Cui, S. A. Cummer, and D. R. Smith, Experimental Demonstration of Electromagnetic Tunneling through an Epsilon-Near-Zero Metamaterial at Microwave Frequencies, Phys. Rev. Lett. 100, 023903 (2008)

[26] Y. Li, B. Liang, Z. Gu, X. Zou, and J. Cheng, Unidirectional acoustic transmission through a prism with near-zero refractive index, Appl. Phys. Lett. 103, 053505 (2013).

[27] Z.-M. Gu, B. Liang, X.-Y. Zou, J. Yang, Y. Li, J. Yang, and J.-C. Cheng, One-way acoustic mirror based on anisotropic zero-index media, Appl. Phys. Lett. 107, 213503 (2015).

[28] C. Shen, Y. Xie, J. Li, S. A. Cummer, and Y. Jing, Asymmetric acoustic transmission through near-zero-index and gradient-index metasurfaces, Appl. Phys. Lett. 108, 223502 (2016).

[29] I. Liberal, A. M. Mahmoud, Y. Li, B. Edwards, and N. Engheta, Photonic doping of epsilon-near-zero media, Science 355, 1058 (2017).

[30] I. Liberal and N. Engheta, Nonradiating and radiating modes excited by quantum emitters in open epsilon-nearzero cavities, Sci. Adv. 2, e1600987 (2016).

[31] V. C. Nguyen, L. Chen, and K. Halterman, Total Transmission and Total Reflection by Zero Index Metamaterials with Defects, Phys. Rev. Lett. 105, 233908 (2010).

[32] Y. Wu and J. Li, Total reflection, and cloaking by zero index metamaterials loaded with rectangular dielectric defects, Appl. Phys. Lett. 102, 183105 (2013).
[33] J. Hao, W. Yan, and M. Qiu, Super-reflection and cloaking based on zero index metamaterial, Appl. Phys. Lett. 96, 101109 (2010).

[34] Y. Xu and H. Chen, Total reflection, and transmission by epsilon-near-zero metamaterials with defects, Appl. Phys. Lett. 98, 113501 (2011).

[35] J. Luo, J. Li, and Y. Lai, Electromagnetic Impurity-Immunity Induced by Parity-Time Symmetry, Phys. Rev. X 8, 031035 (2018).

[36] C. T. Chan, X. Huang, F. Liu, and Z.H. Hang, Dirac dispersion and zero-index in two dimensional and three dimensional photonic and phononic systems, Prog. Electromagn. Res. B 44, 163 (2012).

[37] Z. Li, Z. Liu, and K. Aydin, Wideband zero-index metacrystal with high transmission at visible frequencies, J. Opt. Soc. Am. B 34, D13 (2017).

[38] F. Liu, F. Zhang, W. Wei, N. Hu, G. Deng, and Z. Wang, Scattering of waves by three-dimensional obstacles in elastic metamaterials with zero index, Phys. Rev. B 94, 224102 (2016).

[39] C. He, S.-Y. Yu, H. Ge, H. Wang, Y. Tian, H. Zhang, X.-C. Sun, Y. B. Chen, J. Zhou, M.-H. Lu, and Y.-F. Chen, Threedimensional topological acoustic crystals with pseudospinvalley coupled saddle surface states, Nat. Commun. 9, 4555 (2018).

[40] L. Lu, C. Fang, L. Fu, S. G. Johnson, J. D. Joannopoulos, and M. Soljačić, Symmetry-protected topological photonic crystal in three dimensions, Nat. Phys. 12, 337 (2016).

[41] H.-X. Wang, Y. Chen, Z. H. Hang, H.-Y. Kee, and J.-H. Jiang, 3D Z2 topological nodes in nonsymmorphic photonic crystals: Ultrastrong coupling and anomalous refraction, https://arxiv.org/abs/1608.02437 (2016).

[42] C. Fang and L. Fu, New classes of three-dimensional topological crystalline insulators: Nonsymmorphic and magnetic, Phys. Rev. B 91, 161105 (2015).

[43] J. Luo, Y. Yang, Z. Yao, W. Lu, B. Hou, Z. H. Hang, C. T. Chan, and Y. Lai, Ultratransparent Media and Transformation Optics with Shifted Spatial Dispersions, Phys. Rev. Lett. 117, 223901 (2016).

[44] C. Liu, J. Luo, and Y. Lai, Acoustic metamaterials with broadband and wide-angle impedance matching, Phys. Rev. Mater. 2, 045201 (2018).

[45] K. Sakoda, Optical Properties of Photonic Crystals (Springer Science \& Business Media, Berlin, 2004).

[46] See Supplemental Material at http://link.aps.org/ supplemental/10.1103/PhysRevLett.124.074501 for the splitting of dipolar states with the deform of unit cell, the effective Hamiltonian near $\Gamma$ point, the relationship between Dirac-like linear dispersion and double zero parameters, the effective parameter retrieval method, which includes Refs. [47,48], derivations of transverse acoustic waves as a result of zero effective mass density and the total transmission through a DZIM slab with a finite thickness, the transmission study of PC with single-zero property and double-zero property, the details about the experiments as well as the cloaking effect in the acoustic periscope.

[47] W. Smigaj and B. Gralak, Validity of the effective-medium approximation of photonic crystals, Phys. Rev. B 77, 235445 (2008). 
[48] Z. Yao, J. Luo, and Y. Lai, Illusion optics via onedimensional ultratransparent photonic crystals with shifted spatial dispersions, Opt. Express 25, 30931 (2017).

[49] J. Mei, Y. Wu, C. T. Chan, and Z.-Q. Zhang, First-principles study of Dirac and Dirac-like cones in phononic and photonic crystals, Phys. Rev. B 86, 035141 (2012).

[50] H.-X. Wang, Y. Chen, Z. H. Hang, H.-Y. Kee, and J.-H. Jiang, Type-ii Dirac photons, npj Quantum Mater. 2, 54 (2017).

[51] L.-H. Wu and X. Hu, Scheme for Achieving a Topological Photonic Crystal by Using Dielectric Material, Phys. Rev. Lett. 114, 223901 (2015).

[52] L. Lu, L. Fu, J. D. Joannopoulos, and M. Soljačić, Weyl points, and line nodes in gyroid photonic crystals, Nat. Photonics 7, 294 (2013).

[53] L. Lu, Z. Wang, D. Ye, L. Ran, L. Fu, J. D. Joannopoulos, and M. Soljačić, Experimental observation of Weyl points, Science 349, 622 (2015).

[54] H. He, C. Qiu, L. Ye, X. Cai, X. Fan, M. Ke, F. Zhang, and Z. Liu, Topological negative refraction of surface acoustic waves in a Weyl phononic crystal, Nature (London) 560, 61 (2018).

[55] H. Jia, R. Zhang, W. Gao, Q. Guo, B. Yang, J. Hu, Y. Bi, Y. Xiang, C. Liu, and S. Zhang, Observation of chiral zero mode in inhomogeneous three-dimensional Weyl metamaterials, Science 363, 148 (2019).
[56] B. Yang, Q. Guo, B. Tremain, R. Liu, L. E. Barr, Q. Yan, W. Gao, H. Liu, Y. Xiang, J. Chen, C. Fang, A. Hibbins, L. Lu, and S. Zhang, Ideal Weyl points and helicoid surface states in artificial photonic crystal structures, Science 359, 1013 (2018).

[57] Z. Yang and B. Zhang, Acoustic Type-II Weyl Nodes from Stacking Dimerized Chains, Phys. Rev. Lett. 117, 224301 (2016).

[58] F. Li, X. Huang, J. Lu, J. Ma, and Z. Liu, Weyl points and Fermi arcs in a chiral phononic crystal, Nat. Phys. 14, 30 (2018).

[59] W.-J. Chen, M. Xiao, and C. T. Chan, Photonic crystals possessing multiple Weyl points and the experimental observation of robust surface states, Nat. Commun. 7, 13038 (2016).

[60] J. Li and C. T. Chan, Double-negative acoustic metamaterial, Phys. Rev. E 70, 055602(R) (2004).

[61] Y. Wu, J. Li, Z.-Q. Zhang, and C. T. Chan, Effective medium theory for magnetodielectric composites: Beyond the longwavelength limit, Phys. Rev. B 74, 085111 (2006).

[62] V. C. Henríquez, V. M. García-Chocano, and J. SánchezDehesa, Viscothermal losses in double-negative acoustic metamaterials, Phys. Rev. Applied 8, 014029 (2017).

[63] T. Brunet, A. Merlin, B. Mascaro, K. Zimny, J. Leng, O. Poncelet, C. Aristégui, and O. Mondain-Monval, Soft 3D acoustic metamaterial with negative index, Nat. Mater. 14, 384 (2015). 